\title{
Ceramic-matrix composites take the heat
}

\author{
By Melissae Fellet \\ Feature Editor Wolfgang Rossner
}

$\mathrm{U}$ S air carriers consume tens of billions of gallons of fuel a year. Though their fuel consumption dropped $15 \%$ between 2000 and 2014, rising oil prices meant that fuel costs increased more than $300 \%$ during that time. Even when oil prices drop, airlines still want to improve efficiency.

One way to increase fuel efficiency, and thus decrease fuel costs, is to increase the engine operating temperature. However, temperatures inside current aircraft turbines are already approaching the melting point of the metal superalloys used to make the engine components. These superalloys show surface softening at temperatures $15 \%$ below their melting point; normal materials, on the other hand, show surface softening at temperatures $30 \%$ below their melting temperature.

Engine makers like General Electric (GE) and Rolls-Royce are now replacing some metal parts in their aircraft turbines with ones made from ceramic-matrix composites (CMCs). $\mathrm{CMC}$ components can withstand higher temperatures than ones made from current superalloys. They are also about a third as dense as their metal counterparts, providing additional fuel savings by reducing weight, particularly for rotating parts.

A GE engine with a CMC component will appear on narrow body commercial aircraft starting in 2016. According to the company, this new engine uses $15 \%$ less fuel than its predecessor. Since aircraft turbines are structurally similar to land-based turbines that produce electricity, GE engineers hope to build CMC parts for other turbines too.

Ceramics researchers have dreamed of using CMCs in gas turbines for more than 40 years, said Krishan Luthra, chief scientist for manufacturing, chemical, and materials technologies at GE Global Research. Incremental improvements in metal superalloys have taken about three decades of research to increase turbine operating temperature from $950^{\circ} \mathrm{C}$ to around $1100^{\circ} \mathrm{C}$ today. With $\mathrm{CMC}$ components, engines can run at temperatures closer to $1300^{\circ} \mathrm{C}$, gaining a $150^{\circ} \mathrm{C}$ increase in one step. "That's why they're revolutionary," Luthra said.

Ceramics are typically brittle materials that shatter under stress. Fiber-reinforced CMCs, however, often fail like wood. When stressed, these materials first develop microcracks in the ceramic matrix, then they slowly bend, and eventually, they completely break. Woven fibers in CMCs bridge the microcracks and provide damage tolerance by hindering crack opening and further propagation.
Today's best-performing CMCs are based on siliconcarbide $(\mathrm{SiC})$ constituents. These $\mathrm{SiC} / \mathrm{SiC}$ materials are typically made from nearly polycrystalline $\mathrm{SiC}$ fibers that are first covered with a boron nitride coating and then encased in a $\mathrm{SiC}$ matrix. To make the fibers, manufacturers cure and pyrolyze polymer precursors into long strands of $\mathrm{SiC}$ about 10-15 $\mu \mathrm{m}$ in diameter. Then they spin hundreds of these strands into rope-like tows. These tows are then woven into two-dimensional sheets, like thread is woven into fabric.

Next, individual fibers in the sheets are coated with an interphase material, commonly boron nitride. This coating is important for how CMCs behave under stress. The coating provides a weak interface between the fiber and the matrix so when matrix cracks reach this interface, they travel along and around the fibers, rather than through them.

Once coated, the sheets are stacked in a jig. The fibers in the sheets are aligned to provide maximum strength and stiffness according to the forces the material will experience during use. Materials under one-directional force are constructed so that the majority of their fibers are aligned parallel to that force, while CMCs under fairly uniform stress have fiber directions that alternate between parallel and perpendicular to the force.

Finally, the fiber stack is covered with a ceramic matrix. The components are introduced as gases, polymer solutions, liquid slurries, and/or molten silicon, and the SiC-based ceramic is formed under high temperature. Each processing method creates a material with different porosity. To minimize porosity and maximize performance, different sequences of methods are often used to create the matrix.

Pores reduce the amount of stress a material can handle before the matrix cracks. Under high temperatures, matrix cracks facilitate side reactions that weaken the CMC. Oxygen and water vapor travel through these cracks, attacking the fibers and their coating. These reactions can volatilize the coating and leave a residue that bonds fibers to the matrix or to each other. Oxygen also reacts with silicon or silicon carbide in the matrix, forming oxides on the CMC outer surface that volatilize upon reaction with water vapor produced during combustion. To prevent these detrimental side reactions, many $\mathrm{CMCs}$ are coated with an external oxide-based environmental barrier coating, often based on aluminosilicates.

After fabrication, a CMC is performance tested to see if it can withstand the operating atmosphere, forces, temperatures, 
and pressures without cracking or delaminating. In turbines, the materials need to last for tens of thousands of hours and maintain their durability even if hit with flying debris.

Between 1997 and 2004, Solar Turbines, a California-based gas turbine manufacturer, tested $\mathrm{SiC}$ - and oxide-based $\mathrm{CMC}$ liners in the combustion chamber of their land-based turbines. Oxide-based CMCs contain alumina or mullite fibers in a matrix of alumina, silica, mullite, and/or rare-earth phosphates. They are more stable in oxidizing environments than $\mathrm{SiC} \mathrm{CMCs,}$ making them better suited for the longer lifetimes needed in land-based turbines. But oxide CMCs offer little efficiency gains through temperature increase: they are stable at temperatures similar to current superalloys. After more than 67,000 hours of field tests, the company found that engines containing either type of CMC had lower emissions of carbon monoxide and nitrous oxides than the standard turbine.

GE also tested SiC-based CMC components in land-based electrical turbines, until market forces led them to focus on aviation applications, said Luthra, who has worked on CMCs for about 30 years. GE's new LEAP engine contains a SiC-based CMC shroud that surrounds the turbine's rotating blades. It will be featured first on Airbus A320neo planes and then, in 2017, on Boeing's 737 Max.

Changing the composition of a stationary component also required redesigning the engine. Turbines with metal shrouds are designed so that air passes over the shroud to keep it cool. Since this air does not go through the turbine, it does not provide any extra propulsion. An engine with a CMC shroud, however, needs less cooling air. Redesigning the engine to reduce or eliminate the cooling air also increases its efficiency.

Lessons from using CMCs in aircraft engines will advance GE's efforts to develop CMC components in electrical turbines, Luthra said. Because CMCs last longer than metal, the new components could reduce scheduled turbine maintenance that requires plants to completely shut down. And as with aircraft engines, CMC components could also provide the same improved efficiency and reduced emissions in electrical turbines.

Another frontier for CMC research is a push from National Aeronautics and Space Administration (NASA) scientists to increase the material's temperature stability to near $1500^{\circ} \mathrm{C}$. "Going to higher temperatures means better fibers, and we don't have them yet," said James DiCarlo, who has worked on CMCs at NASA Glenn Research Center for more than 35 years.

The goal is to develop fibers that resist creep, a deformation property that impacts the microstructure of the fiber, particularly at high temperatures. During creep, the fiber lengthens, grains in the polycrystalline fiber slide past each other, and holes appear between the grains. Those holes can eventually result in fracture of the fiber when a CMC is under stress. Creep also reduces a fiber's ability to bridge growing matrix cracks.

To improve the fibers, NASA scientists are attempting to alter the microstructure of commercially available fibers by tailoring the grain size and atomic composition to reduce creep. The group is also working to improve CMC toughness by interlacing the fibers in three-dimensional patterns, so that some fibers wind up and down through the thickness of a stack of 2D sheets. One challenge with this architecture is that the fibers rub against each other during weaving and can weaken from the abrasion. "There's still research to be done: better fibers, better architectures, better ways of making fibers into those architectures, better matrix," DiCarlo said. CMC commercialization could help researchers improve the materials and develop them for other applications. Currently, CMCs are more expensive, and less predictable, in terms of performance, than metal superalloys. Only a few Japanese companies produce SiC fibers, in small amounts and with varying consistency. "The amount of fibers that GE needs to make all these parts will probably change everything," said Gregory N. Morscher, at The University of Akron. Now that GE is optimizing their material and scaling up production to fill about 9600 orders for the LEAP engine, commercial fibers may have more consistent properties. "It's still expensive, but things are getting cheaper and more reproducible."

For CMC researchers and airline enthusiasts, the coming years will be exciting as more CMC turbine components appear on the market. Engineers at Boeing flight-tested an engine with a CMC nozzle in September 2014 as part of a federal program to improve aircraft fuel efficiency and reduce noise and emissions. In March 2015, GE groundtested a commercial turbine with multiple CMC components. The GE9X, developed for the Boeing 777X and expected to be released in 2020, will have CMC nozzles, combustion liners, and turbine shrouds. The company is also developing a turbine for military aircraft that has rotating CMC turbine blades.

Triplicane (Tap) Parthasarathy, director of materials and processes at the research and development company UES Inc., Dayton, Ohio, USA, who has worked on CMCs for almost 30 years said, "It's not often in a scientist's career that something you work on will be used somewhere that benefits humanity at large." $\square$ 
